\title{
BMJ Open Complex social intervention for multidisciplinary teams to improve patient referrals in obstetrical care: protocol for a stepped wedge study design
}

\author{
Anita Romijn, ${ }^{1}$ Martine C de Bruijne, ${ }^{1}$ Pim W Teunissen, ${ }^{2}$ \\ Christianne J M de Groot, ${ }^{2}$ Cordula Wagner ${ }^{1,3}$
}

To cite: Romijn A, de Bruijne MC, Teunissen PW, et al. Complex social intervention for multidisciplinary teams to improve patient referrals in obstetrical care: protocol for a stepped wedge study design. BMJ Open 2016;6: e011443. doi:10.1136/ bmjopen-2016-011443

- Prepublication history for this paper is available online. To view these files please visit the journal online (http://dx.doi.org/10.1136/ bmjopen-2016-011443).

- Additional material is published online only. To view, please visit the journal online (http://dx.doi.org/10. 1136/bmjopen-2016-011443)

Received 8 February 2016 Revised 17 May 2016 Accepted 19 May 2016

CrossMark

For numbered affiliations see end of article.

Correspondence to Anita Romijn; a.romijn@vumc.nl

\section{ABSTRACT}

Introduction: In obstetrics, patients often experience referral situations between different care professionals. In these multidisciplinary teams, a focus on communication and interprofessional collaboration is needed to ensure care of high quality. Crew resource management team training is increasingly being applied in healthcare settings to improve team performance and coordination. Efforts to improve communication also include tools for standardisation such as SBAR (situation, background, assessment, recommendation). Despite the growing adoption of these interventions, evidence on their effectiveness is limited, especially on patient outcomes. This article describes a study protocol to examine the effectiveness of a crew resource management team training intervention aimed at implementing the SBAR tool for structured communication during patient referrals in obstetrical care.

Methods and analysis: The intervention is rolled out sequentially in five hospitals and surrounding primary care midwifery practices in the Netherlands, using a stepped wedge design. The intervention involves three phases over a period of 24 months: (1) preparation, (2) training and (3) follow-up with repeated measurements. The primary outcomes are perinatal and maternal outcomes calculated using the Adverse Outcome Index. The secondary outcomes are the reaction of participating professionals to the training programme, attitudes towards safety and teamwork (Safety Attitudes Questionnaire), cohesion (Interprofessional Collaboration Measurement Scale), use of the tool for structured communication (selfreported questionnaire) and patient experiences. These secondary outcomes from professional and patient level allow triangulation and an increased understanding of the effect of the intervention on patient outcomes.

Ethics and dissemination: The study was approved by the Medical Ethical Committee of the VU University Medical Centre in the Netherlands and the protocol is in accordance with Dutch privacy regulations. Study findings will be presented in publications in peer-

\section{Strengths and limitations of this study}

- Multiple measures at professional and patient level and a long follow-up period form the basis of a rigorous evaluation process.

- In a multicentre study, the intervention can be implemented sequentially because of the stepped wedge design which also enables us to take intervention and control conditions into account in the analyses.

- Limited measures are available to gain insight into behavioural change within obstetrical teams.

- This study is conducted in a dynamic context for research considering the variability of local practices and organisation of obstetrical care in the Netherlands.

reviewed journals and presentations at scientific conferences.

Trial registration number: NTR4256; Pre-results.

\section{INTRODUCTION}

Differences in perinatal mortality and morbidity rates indicate a significant variation in the quality of obstetrical care between European countries. In 2010, the highest fetal mortality rates (at or after 28 weeks of gestation) were almost three times higher than the lowest rates in Europe. ${ }^{1}$ The international comparison indicated that a reduction in adverse obstetrical health outcomes is possible in countries with high as well as low perinatal mortality rates. ${ }^{12}$ When considering causes of adverse health outcomes in obstetrics, studies have shown that about $10-60 \%$ are associated with suboptimal care. ${ }^{23} \mathrm{~A}$ main cause of suboptimal care is poor communication between care professionals. $^{4-6}$ Therefore, a focus on 
interprofessional communication is indispensable if the quality of obstetrical care is to be improved. ${ }^{7}$

Four decades ago, deficiencies in communication and collaboration were found to be important contributing factors in major accidents in aviation. As a result, specialised training programmes were developed, such as crew resource management (CRM), in an effort to improve critical safety behaviours. CRM focuses on team performance and team coordination. Performance limiters are considered during team training sessions, as well as the nature and detection of human error and countermeasures to deal with errors. ${ }^{8} 9$ In 2000, the Institute of Medicine advocated the adoption of aviation's approach to safety and error management in healthcare. ${ }^{10}$ CRM team training is now increasingly being applied in various healthcare settings, including obstetrical care. ${ }^{11-14}$

In obstetrics, care is provided by a multidisciplinary team. As a result, patients often experience referrals between different care professionals. Referral situations have been identified as an important risk factor for deficits in communication. ${ }^{15}{ }^{16}$ Information might not be passed on or may be misunderstood by care providers during the referral. ${ }^{15}$ Efforts to improve communication, in addition to CRM team training, include tools for standardisation such as SBAR (situation, background, assessment, recommendation). SBAR provides a common and predictable structure for the communication of information that covers variations in communication styles and helps to create a shared mental model between care providers. ${ }^{17} 18$ The use of the SBAR structure during referral situations is aimed to improve the transfer of clinical and procedural information and team performance. ${ }^{19}$

Despite the growing adoption of CRM team training and SBAR in healthcare settings, evidence on the effectiveness of these interventions is limited. ${ }^{14}{ }^{20}$ So far, results indicate that classroom-based CRM team training has had positive effects on participants' reactions and attitudes towards teamwork and safety. ${ }^{12}{ }^{21}$ In addition, the use of SBAR seems to improve interprofessional collaboration and communication. ${ }^{17} 22$ However, results for the impact of CRM team training and SBAR on learning and behavioural changes are mixed, and there is a lack of information on effects on patient outcomes. ${ }^{21}{ }^{22}$ Previous studies often only use pre-post measurements, include a short follow-up period and restrict their intervention to one department or setting. A need remains for studies with multiple measures, including patient outcomes. $^{12} 2123$ To obtain evidence on the impact of CRM team training and SBAR interventions, it requires a complex intervention study with an explicit focus on interprofessional communication and collaboration. ${ }^{23}$

These recommendations from previous research are incorporated in the study protocol of the Local Obstetrical Collaboration Multidisciplinary Onsite Teamtraining effectiveness (LOCoMOTive) study. This study is designed to examine the effectiveness of an intervention based on CRM team training that is aimed at implementing the SBAR tool for structured communication during patient referrals in obstetrical care. The intervention is a combination of CRM team training and SBAR, two complementary methods to improve communication and collaboration. It is a multicentre study in a complex setting with multiple care providers and a high patient referral rate between echelons. ${ }^{24}{ }^{25}$ We aim to study the effect of the intervention on adverse patient outcomes using a stepped wedge design. In addition, secondary outcome measures from professional and patient levels are included. By collecting data on multiple measures over a long follow-up period, we aim to increase understanding of the implementation and impact of CRM team training and SBAR interventions. The purpose of this paper is to describe the study protocol for the LOCoMOTive study in detail.

\section{METHODS AND ANALYSIS \\ Setting}

In the Netherlands, obstetrical care is provided by different groups of professionals working in three echelons: primary, secondary and tertiary care. Primary care midwives take care of women with low risks of pathology. For these women, care is provided by independent midwifery practices in the locality. Primary care midwives refer women to a secondary care hospital if risks of adverse fetal or maternal outcomes are high or if complications arise during pregnancy or childbirth. Risk selection, based on a standard list of indications to consult or refer women to a higher echelon, forms the basis of this system. ${ }^{24}$

In secondary care hospitals, gynaecologists work together with paediatricians, clinical midwives, nurses and specialist registrars to provide the appropriate level of care. More specialised care is provided in academic hospitals, representing the third echelon. In these hospitals, care can also be provided for more complex and acute cases, including (suspected) preterm births before 32 weeks of gestation. ${ }^{24} 25$ Besides clinical relevance, there are elective reasons for referring women from primary to secondary care, such as analgesia during childbirth. A consequence of this set-up is that almost $50 \%$ of all pregnant women in the Netherlands are referred from primary to secondary or tertiary obstetrical care during pregnancy or childbirth. ${ }^{25} 26$ The intervention described in this study protocol focuses primarily on these patient referrals between echelons.

The north-western region of the Netherlands includes a population of around 2.7 million people, with a total of just over 29000 births per year. ${ }^{27} 28$ In this region, obstetrical care is provided by 18 local obstetrical collaborations (LOCs). An LOC refers to a hospital (secondary or tertiary care) and the surrounding primary care midwifery practices that are their preferred referring practices. In 2013, the 18 LOCs in this region started a collaborative perinatal network, as part of a national 'Pregnancy and Childbirth' programme. The aim of this programme is to improve perinatal and maternal outcomes by means of innovation and research projects. ${ }^{29}$ 
The LOCoMOTive study, conducted from January 2013 to December 2016, is one of the research projects assigned to the Regional Perinatal Network.

\section{Study population}

The 18 LOCs in the north-western region of the Netherlands were all informed about the study and invited to take part. Inclusion criteria for LOCs were interest in participation, sufficient support from all the parties involved (ie, hospital staff in the obstetrics department and primary care midwives associated with the LOC), willingness to provide insight into data on perinatal morbidity drawn from the Dutch Perinatal Registry, readiness to take part in the 24-month intervention period (including implementation and follow-up phase) and agreement to a procedure of random assignment to an intervention period, knowing it could possibly take a year before they would start with the intervention.

Ten LOCs met these requirements; however, only five LOCs could be included in the study. The inclusion of more than five LOCs was not feasible given the constraints in resources and the desire to maintain a high quality in the intervention and have a sound evaluation process. A random selection procedure was used to select 5 out of the 10 LOCs, taking into account the type of hospital (secondary and tertiary care) allocated to an LOC. The five selected LOCs were then randomly assigned to an intervention period using an online randomisation tool. In total, 467 care professionals participate in the study, including primary care midwives $(n=124)$, clinical midwives $(n=44)$, gynaecologists and registrars in gynaecology $(\mathrm{n}=84)$ and nurses $(\mathrm{n}=215)$.

\section{Design}

A stepped wedge design is used in which the intervention of the LOCoMOTive study is rolled out sequentially in the different LOCs. The intervention is described in detail in the next section. A stepped wedge design is a type of cross-over design in which different clusters switch from control to intervention conditions at different time points. ${ }^{30}$ In the LOCoMOTive study, the five LOCs in the north-western region of the Netherlands represent the different clusters. All obstetrical care professionals working in the LOCs receive the intervention; however, the order in which the LOCs participate is determined at random. ${ }^{31}{ }^{32}$ In the LOCoMOTive study, a new LOC starts with the intervention and follow-up period at three-monthly time intervals, as described below.

\section{Intervention}

The intervention involves the implementation of a structured referral procedure using SBAR, introduced by multidisciplinary classroom-based CRM team training. This intervention primarily focuses on the patient referrals between primary care and secondary or tertiary care. Critical success factors for team training in healthcare, as defined by Salas, are taken into account. ${ }^{33}$ They entail alignment of intervention objectives with organisational goals, inventory of the required resources and time commitment and an effort to ensure their availability, involvement of front-line care leaders, providing organisational support, adequate preparation of the environment and participants for the intervention, facilitation of a training programme reflecting real-time practice and evaluation of the effectiveness of the intervention. ${ }^{33}$ The intervention period consists of three phases with a total period of 24 months per LOC. A diagram of the intervention period is shown in figure 1 .

\section{Preparation phase (3 months)}

In the first 3 months of the intervention, a multidisciplinary project team is formed to support the intervention period in their LOC. This team consists of at least a primary care midwife, a clinical midwife, a nurse, a registrar in obstetrics and gynaecology and a gynaecologist. During the preparation phase, the planning of the intervention is discussed with this team, taking the local situation with regard to the critical success factors into account. Moreover, the project team discusses operationalisation of specifics of the patient population and measurements and schedules the team training sessions. Finally, baseline measurements are performed. These are described in the next section.

\section{Training phase (3 months)}

The classroom-based CRM team training consists of two 3-hour sessions, about 4 weeks apart, in multidisciplinary groups with a maximum of 20 obstetrical care
Figure 1 Diagram of the phases and measurements of the intervention period per local obstetrical collaboration (LOC). A new LOC starts every 3 months with the same intervention scheme.

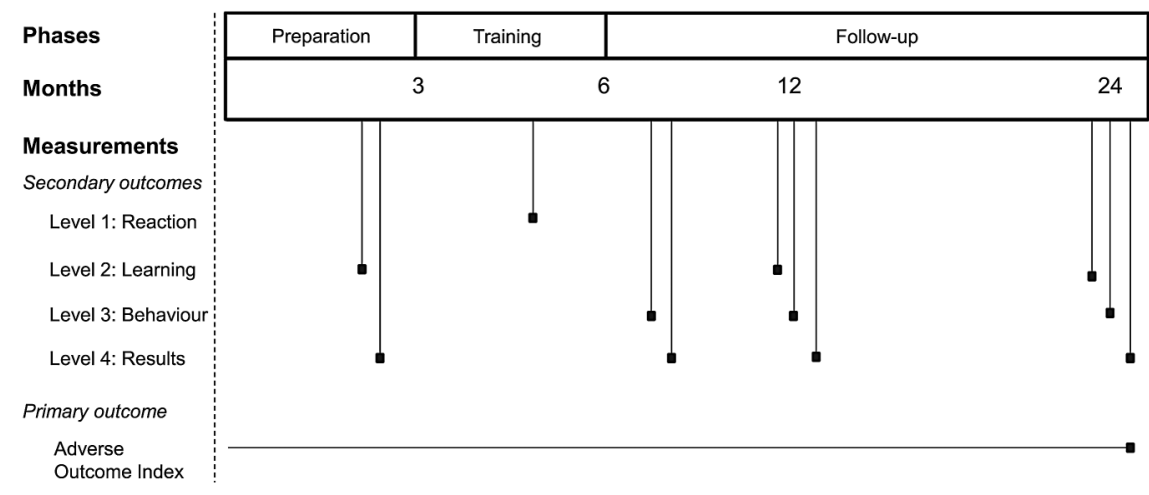


professionals. In order to include the entire team of obstetric professionals per LOC, multiple sessions are scheduled for the two parts of the training. In total, 49 team training sessions are organised. The training serves a dual purpose: (1) to develop interpersonal knowledge and skills needed to collaborate optimally and manage resources within the chain of obstetrical care; (2) to improve interprofessional communication during patient referrals by using SBAR for a structured communication procedure. In addition to the SBAR structure, the team training covers reading back by the recipient of the information for verification. The training is highly interactive and includes role-playing reflecting real referral situations. Table 1 provides an overview of the programme for the two parts of the team training. Furthermore, accreditation for participation was obtained from the professional bodies of midwives, nurses and gynaecologists.

\section{Follow-up phase (18 months)}

After the multidisciplinary training sessions, LOCs are monitored during 18 months. The implementation of the structured referral procedure is supported using a multifaceted strategy, including feedback and reminders. ${ }^{34}$ In addition, the project team continues to discuss barriers and facilitators for implementation and is responsible for motivating the care professionals in the LOC to make use of the procedure for structured referrals. This set-up lets the LOCs organise the implementation and related actions to their liking, which may lead to variations between the five LOCs. Follow-up measurements are performed at 8,12 and 24 months into the intervention period.

\section{Primary outcome measure}

The main objective of the LOCoMOTive study is to assess the effect of the CRM team training intervention, aimed at implementing the SBAR tool during patient referrals, on perinatal and maternal outcomes. The patient outcomes are assessed using compound measures drawn from the Dutch Perinatal Registry. This medical registry includes data from the registries of primary midwives, general practitioners, obstetricians, paediatricians and neonatologists. These combined data provide insight into perinatal care, processes and outcomes per LOC. ${ }^{26}$ As individual adverse events are rare, obstetrical outcome measures were developed by Mann et $a l^{35}$ The Adverse Outcome Index (AOI) was developed to measure the frequency of deliveries with one or more adverse event. ${ }^{35} 36$ The AOI is the primary dependent variable in this study.

The AOI is defined by the following poor clinical outcomes related to the fetus or neonate: intrapartum death of a fetus weighing at least $500 \mathrm{~g}$ at 24 weeks of gestation or greater; neonatal death of a baby with a birth weight of $2500 \mathrm{~g}$ or more within 7 days of birth; neonatal birth trauma (Erb's palsy or a vacuum or forceps injury noted within 24 hours of birth) in a fetus of at least 20 weeks of gestation; unplanned admission of a term neonate (birth weight $2500 \mathrm{~g}$ or more and

Table 1 Overview of the programme for the classroom-based crew resource management (CRM) team training sessions

\begin{tabular}{|c|c|c|}
\hline Themes & Objectives & Methods \\
\hline $\begin{array}{l}\text { Crew resource } \\
\text { management }\end{array}$ & $\begin{array}{l}\text { To explain the background to increasing attention } \\
\text { for non-technical skills in teams and the related } \\
\text { translation from aviation to a healthcare setting }\end{array}$ & Plenary session \\
\hline $\begin{array}{l}\text { Situational } \\
\text { awareness (SA) }\end{array}$ & $\begin{array}{l}\text { To explain what SA is, how it can be established, } \\
\text { what SA means in practice and the risks } \\
\text { associated with a loss of SA }\end{array}$ & $\begin{array}{l}\text { Plenary session, supported by images to enhance } \\
\text { interpretation }\end{array}$ \\
\hline Group vs team & $\begin{array}{l}\text { To clarify the differences between groups and } \\
\text { teams and to underline the importance of team } \\
\text { effort and working towards a common goal }\end{array}$ & Group discussion \\
\hline $\begin{array}{l}\text { Theory of James } \\
\text { Reason }\end{array}$ & To address error causation and error management & $\begin{array}{l}\text { Plenary session and group assignment to apply } \\
\text { the theory to an example from everyday practice }\end{array}$ \\
\hline Hierarchy & $\begin{array}{l}\text { To address (negative) effects of hierarchy within an } \\
\text { organisation and to discuss possible issues } \\
\text { caregivers experience within their team }\end{array}$ & Group discussion \\
\hline Referral procedure & $\begin{array}{l}\text { To discuss the current referral procedure between } \\
\text { primary care midwives and secondary or tertiary } \\
\text { caregivers and to introduce a structured procedure } \\
\text { for referral, using SBAR and a read back }\end{array}$ & $\begin{array}{l}\text { Plenary session, group discussion and } \\
\text { assignments, practising SBAR and reading back } \\
\text { of information for verification in referral situations }\end{array}$ \\
\hline Implementation & $\begin{array}{l}\text { To provide tips and discuss ways to implement } \\
\text { SBAR in everyday practice }\end{array}$ & Group discussion \\
\hline $\begin{array}{l}\text { Improvement } \\
\text { efforts }\end{array}$ & $\begin{array}{l}\text { To incorporate the various themes addressed in } \\
\text { the two team training sessions into personal and } \\
\text { team efforts to improve care }\end{array}$ & $\begin{array}{l}\text { Group discussion and assignment, to sum up } \\
\text { improvement efforts }\end{array}$ \\
\hline
\end{tabular}


gestational age 37 weeks or more) to neonatal intensive care within 24 hours of birth for 24 hours or more; 5 min Apgar score of $<7$ in a neonate with a birth weight of $2500 \mathrm{~g}$ or more. In addition, the maternal adverse events included are maternal death; uterine rupture; unplanned admission to intensive care; unplanned return to the obstetrical unit or to the operating room; blood transfusion; presence of a third-degree or fourthdegree laceration during vaginal delivery. ${ }^{36}$

In addition to the AOI, the Weighted Adverse Outcome Score and the Severity Index are used to provide an assessment of the severity, rather than the frequency, of adverse events. The Weighted Adverse Outcome Score assigns a weight to the outcome, ranging from 5 (third-degree or fourth-degree perineal tear) to 750 (maternal death). The score assigned to a delivery is the sum of weights for each adverse outcome that occurred during a delivery, or zero if no adverse outcomes occurred. The Weighted Adverse Outcome Score is then defined as the total weighted score of each adverse outcome divided by the total number of deliveries. Finally, the Severity Index captures the severity of the types of poor outcomes for those patients with one or more adverse outcomes. This index is calculated by the total weighted score divided by the total number of deliveries with one or more adverse outcomes. ${ }^{35} 36$

In accordance with Mann et al, we use similar definitions of adverse events and poor clinical outcomes. There is no research available on the prevalence of the AOI before the intervention in the LOCs, but we estimate this to be $\sim 5 \%$. For effects in an individual LOC with 2000 deliveries preintervention and postintervention, the study will have a power of $90 \%$ to yield a significant effect $(\alpha 0.05)$, when the intervention decreases the prevalence of the AOI by $2 \%$. For the multicentre effect, when including 6000 deliveries preintervention and postintervention, the study will have a power of $80 \%$ to yield a significant effect, when the intervention decreases the prevalence of the AOI by $1 \%$. A Dutch version of the AOI based on the Dutch Perinatal Registry is being operationalised at the time of writing. We will use the definitive Dutch version for the analyses of the three obstetrical outcome measures in the LOCoMOTive study.

\section{Secondary outcome measures}

The intervention could affect the obstetrical care in the LOCs at different levels. Therefore, to assess effects on aspects other than adverse patient outcomes, we use the training evaluation model of Kirkpatrick. ${ }^{37}$ This model describes four levels of outcomes from an intervention: (1) Reaction-to what degree are participants satisfied with the training? (2) Learning - to what degree do participants acquire the intended knowledge, skills and attitudes? (3) Behaviour-to what degree do participants apply what they learnt during training? (4) Results-to what degree do targeted outcomes improve as a result of the training?
The organisational structure of obstetrical care in the Netherlands, the complexity of the intervention and time limitations have forced us to choose a limited number of outcomes per level. Nonetheless, it is important to measure outcomes at all four levels because this allows triangulation and can potentially lead to extra insights in addition to the primary outcome. For instance, failure to discover behavioural changes (level 3) may be the result of insufficient implementation of SBAR. However, if the finding for levels 1 and 2 indicate that participants positively assessed the intervention and that attitudes towards collaboration, teamwork and safety have improved due to the intervention, then failure to achieve behavioural changes at level 3 may reflect shortcomings in other preconditions such as organisational or managerial support. The measurements for each level are described below.

\section{Level 1: participants' reaction}

The measurement at this level involves the assessment of the reaction of participant to the training programme using a self-reported questionnaire. After the second session of the multidisciplinary team training, all participants are asked to complete a 10-item questionnaire. The questionnaire assesses the trainers, perceived strong and weak elements of the training, the added value for everyday practice and the applicability of the content, especially regarding the use of the SBAR tool in practice during patient referrals.

\section{Level 2: learning}

Outcomes at this level relate to changes in attitudes towards safety and teamwork as well as perceptions regarding collaboration within the LOCs. To assess these outcomes, we use two validated questionnaires. Both questionnaires were translated into Dutch using consensus meetings with three independent forward translators. Participants are asked to fill in both questionnaires at baseline (during the preparation phase) and at 12 and 24 months into the intervention period. At every measurement period, we include all care professionals who are working at that moment in the obstetrical departments and primary care midwifery practices in the participating LOCs.

First, we use the Safety Attitudes Questionnaire (SAQ - safety and teamwork climate version) to measure care professionals' attitudes to patient safety culture regarding safety and teamwork in their LOC. ${ }^{38}$ In the SAQ teamwork is assessed as the perceived quality of collaboration between caregivers, and safety is related to perceptions of a strong and proactive organisational commitment to safety. ${ }^{38}$ The safety and teamwork climate version has 27 questions using a five-point Likert scale. The questions have been slightly adapted to fit the obstetrical organisation of an LOC.

Second, cohesion among care professionals within the LOCs is assessed using the InterProfessional Collaboration Measurement Scale (IPCMS). Kenaszchuk 
and colleagues developed a 13-item questionnaire using a four-point Likert scale. The questions in the IPCMS relate to communication, accommodation and isolation and are suitable for multiple health provider groups, using a round-robin design. ${ }^{39}$ This means that all the health professional groups involved assess all the other health professional groups on their interprofessional collaboration. In the LOCoMOTive study, the care professionals involved are divided into four groups: gynaecologists (including the registrars), clinical midwives, primary care midwives and nurses. Thus, to measure cohesion, each group assesses the collaboration with the three other groups, completing the questionnaire three times.

\section{Level 3: behaviour}

This level covers behavioural change associated with application of the knowledge and skills acquired during training in everyday practice. Therefore, we measure to what extent the procedure for structured referrals between obstetrical care professionals is used in the LOCs, using a self-reported questionnaire. A 14-item questionnaire was developed to compare the use of SBAR by 'the sender' and 'the recipient' of information. In the LOCoMOTive study, 'the sender' refers to the primary care midwifes and 'the recipients' are the gynaecologists, registrars and clinical midwifes. Information is gained on the perceived frequency of SBAR usage during referrals, the order in which information is presented, perceived ease of use and whether supplementary questions are asked during the referral. The questionnaire uses a five-point Likert scale. Participants are asked to fill in this questionnaire at 8 months (2 months after the CRM team training sessions) and 12 and 24 months into the intervention period.

\section{Level 4: results-patient experiences}

If there is a structured exchange of relevant patient information, the obstetrical team should be better able to respond to specific patient characteristics or preferences. Therefore, measurement of patient experiences is also included in this study. The experiences are assessed using the ReproQ questionnaire. ${ }^{40}$ The postnatal ReproQ questionnaire has 76 questions covering 10 domains referring to the care process, outcomes and experiences with care. For 1 month during the preparation phase, patients who have been referred from primary care to secondary or tertiary care are asked to complete the questionnaire on their experiences during the referral and the cooperation between their caregivers. This procedure is repeated at 8,12 and 24 months into the intervention period.

\section{Statistical analyses}

The primary outcomes are the perinatal and maternal mortality and morbidity rates using the AOI and, in addition, the Weighted Adverse Outcome Score and Severity Index. We hypothesise that improved communication and collaboration in the LOCs due to using SBAR during patient referrals will reduce adverse patient outcomes. This analysis involves comparison of (1) the AOI during the period in which LOCs were allocated to the control conditions with (2) the AOI during the period after LOCs had received the multidisciplinary CRM team training sessions. By using the stepped wedge design, the 3-month time interval of the start of the intervention period for LOCs has to be taken into account when calculating the three obstetrical outcome measures for the control period and intervention period.

The design of this study also allows for pre-post analyses of the secondary outcomes on professional and patient levels. The data collected for the four levels of Kirkpatrick's evaluation model will be checked for completeness and missing data. Imputation of missing data will be applied when appropriate. Descriptive data analyses will be used to study and compare characteristics of the patients and care professionals within and between the five LOCs, before and after the intervention. Characteristics of the care professionals include gender, age, parenthood, years of work experience in their current profession and years in the obstetrical department in the hospital or primary midwifery practice. Patient characteristics include age, parity, gravidity, medical interventions during childbirth and childbirth outcomes. We will decide on which covariates to include in the analyses based on descriptive analyses and literature. Clustering will be taken into account using multilevel analyses when appropriate. The results of pre-post analyses will be used to increase understanding of the effect of the intervention on patient outcomes.

\section{Ethics and dissemination}

The study was approved by the Medical Ethical Committee of the VU University Medical Centre in the Netherlands and the protocol is in accordance with Dutch privacy regulations. The study is registered in the Dutch Trial Registry, record NTR4256. The participating hospitals received approval from their local boards. Moreover, all obstetrical departments and independent midwifery practices gave consent to use data from their medical registries for the analyses of the three obstetrical outcome measures. In addition, informed consent was obtained from all patients who filled in the ReproQ questionnaire. All data will only be used for this study and are stored in anonymised files, which are only accessible to the involved researchers. Study findings will be presented in publications in peer-reviewed journals and presentations at national and international scientific conferences.

\section{DISCUSSION}

This paper described the study protocol of a stepped wedge study on the effectiveness of classroom-based CRM team training aimed at implementing the SBAR tool for structured communication in obstetrical care. 
Improving interprofessional collaboration and communication is indispensable when trying to improve the quality and safety of care. Especially settings which involve complex social networks of individuals with different backgrounds, training, viewpoints and locations of work influence effective communication and teamwork. ${ }^{5}{ }^{17}$ This certainly applies to obstetrical care, where care providers from various backgrounds work in different echelons. We hypothesise that improved collaboration and communication between obstetrical care professionals during patient referrals will reduce adverse patient outcomes.

\section{Strengths and limitations}

This study protocol describes a complex social intervention. Evaluation of the effects of this intervention requires multiple measures, especially since the primary outcomes are patient outcomes. ${ }^{41}$ Since multiple measures and the long follow-up period represent a rigorous evaluation process, the stepped wedge design is appropriate to use for scientific and practical reasons. The stepped wedge design enables intervention and control conditions to be taken into account, concerning adverse patient outcomes in the LOCs. In addition, the complex intervention can be implemented sequentially in the five LOCs. ${ }^{31} \quad 32 \quad 42$ Furthermore, we incorporated Kirkpatrick's model to evaluate additional effects of the intervention from professional and patient levels. The mixed-methods approach allows triangulation, which strengthens the evidence gained from the study. The multiple outcome measures can lead to valuable insights in addition to the primary outcome measure, although this should be carefully evaluated taking the risk of a false-positive effect into account.

The intervention in the LOCoMOTive study requires organisational and behavioural change. In order to be effective, local practice must be taken into account in the execution of the intervention, especially during the follow-up phase. ${ }^{43}$ To support the intervention in the LOCs, we incorporated critical success factors for team training in the study protocol. ${ }^{33}$ Consequently, while the multiple measures and measurements are fixed, the variability in the local context of the LOCs will lead to small variations in the execution of the study. In collaboration with the local project teams, we adapt the implementation of SBAR during patient referrals to suit local practice aiming to achieve a lasting change.

A limitation of this study protocol is the limited availability of measures to evaluate the effects of the intervention. For instance, the variability in local contexts forced us to develop a self-reported questionnaire to evaluate behavioural change. Initially, we aimed to measure the use of SBAR in the LOCs by using telephone recordings of patient referrals. This method was previously used in a simulation setting by Cunningham et al. ${ }^{44}$ However, the application of telephone recordings in everyday practice could not be achieved within the time frame of this study because of practical and legal restrictions.
The privacy of care professionals and patients could not be guaranteed without informed consent, and the different telephone systems used in the LOCs would limit the comparability of results. Yet, taking our resources into account, we argue that we have included methods that are appropriate for the evaluation of the effects on patient outcomes and at all four levels of Kirkpatrick's evaluation model.

The organisation of obstetrical care in the Netherlands is a dynamic context for research. It involves diverse multidisciplinary teams, a high number of patient referrals between primary and secondary or tertiary care and variations in local practice between LOCs. In the LOCoMOTive study, the variability in local context is taken into account. Furthermore, the study builds on previous research to acquire evidence on CRM team training and SBAR interventions and it aims to contribute to the improvement of obstetrical care in the Netherlands. This study protocol is an example of a state-of-the-art evaluation model for a complex social intervention, giving results that will reflect real-life practice.

\section{Author affiliations}

${ }^{1}$ Department of Public and Occupational Health, EMGO+ Institute for Health and Care Research, VU University Medical Center, Amsterdam, The Netherlands

${ }^{2}$ Department of Obstetrics and Gynaecology, VU University Medical Center, Amsterdam, The Netherlands

${ }^{3}$ NIVEL, Netherlands Institute for Health Services Research, Utrecht, The Netherlands

Contributors AR drafted the manuscript. MdB, PT, CdG and CW were involved in the design of the study, drafted the research proposal and participated in the writing process of this manuscript. All authors read and approved the final manuscript.

Funding This work was supported by ZonMw, the Dutch Organisation for Health Research and Development-grant number 209020001. ZonMw had no role in the design of this study and will not have any role during its execution, analysis and interpretation of results.

Competing interests None declared.

Ethics approval Medical Ethics Review Committee of the VU University Medical Center.

Provenance and peer review Not commissioned; externally peer reviewed.

Open Access This is an Open Access article distributed in accordance with the Creative Commons Attribution Non Commercial (CC BY-NC 4.0) license, which permits others to distribute, remix, adapt, build upon this work noncommercially, and license their derivative works on different terms, provided the original work is properly cited and the use is non-commercial. See: http:// creativecommons.org/licenses/by-nc/4.0/

\section{REFERENCES}

1. Euro-Peristat project with SCPE and Eurocat. European Perinata Health Report: Health and care of pregnant women and babies in Europe in 2010. 2013. http://www.europeristat.com

2. Flenady V, Middleton P, Smith GC, et al. Stillbirths: the way forward in high-income countries. Lancet 2011;377:1703-17.

3. Richardus JH, Graafmans WC, Verloove-Vanhorick SP, et al., EuroNatal International Audit Panel; EuroNatal Working Group. Differences in perinatal mortality and suboptimal care between 10 European regions; results of an international audit. BJOG 2003;110:97-105.

4. White AA, Pichert JW, Bledsoe $\mathrm{SH}$, et al. Cause and effect analysis of closed claims in obstetrics and gynecology. Obstet Gynecol 2005;105:1031-8. 
5. Leonard M, Graham S, Bonacum D. The human factor: the critical importance of effective teamwork and communication in providing safe care. Qual Saf Health Care 2004;13(Suppl 1):i85-90.

6. Miller LA. Patient safety and teamwork in perinatal care-Resources for Clinicians. J Perinat Neonat Nurs 2005;19:46-51.

7. American College of Obstetricians and Gynecologists Committee on Patient Safety and Quality Improvement. ACOG Committee Opinion No. 447: Patient safety in obstetrics and gynaecology. Obstet Gynecol 2009;114:1424-7.

8. Helmreich RL. On error management: lessons learned from aviation. $B M J$ 2000;320:781-5.

9. Salas E, Burke SC, Bowers CA, et al. Team training in the skies: does crew resource management (CRM) training work? Hum Factors 2001;43:641-74.

10. Kohn LT, Corrigan JM, Donaldson MS, eds. To err is human: building a safer health system. Washington DC: National Academies Press, 1999.

11. Oriol MD. Crew resource management: applications in healthcare organizations. J Nurs Adm. 2006;36:402-6.

12. Salas E, Wilson KA, Burke SC, et al. Does crew resource management training work? An update, an extension, and some critical needs. Hum Factors 2006;48:392-412.

13. Merién AE, van de Ven J, Mol BW, et al. Multidisciplinary team training in a simulation setting for acute obstetric emergencies; a systematic review. Obstet Gynecol 2010;115:1021-31.

14. Haller G, Garnerin P, Morales MA, et al. Effect of crew resource management training in a multidisciplinary obstetrical setting. Int J Qual Health Care 2008;20:254-63.

15. WHO collaborating centre for patient safety solutions. Communication during patient hand-overs. Patient Saf Solut 2007;1(3).

16. Pezzolesi C, Schifano F, Pickles J, et al. Clinical handover incident reporting in one UK general hospital. Int J Qual Health Care 2010;22:396-401.

17. Marshall S, Harrison J, Flanagan B. The teaching of a structured tool improves the clarity and content of interprofessional clinical communication. Qual Saf Health Care 2009;18:137-40.

18. Guise JM, Segel S. Teamwork in obstetric critical care. Best Pract Res Clin Obstet Gynaecol 2008;22:937-51.

19. Haig KM, Sutton S, Whittington J. SBAR: a shared mental model for improving communication between clinicians. Jt Comm J Qual Patient Saf 2006;32:167-75.

20. Buljac-Samardzic M, Dekker-van Doorn CM, van Wijgaarden JD, et al. Interventions to improve team effectiveness: a systematic review. Health Policy 2010;94:183-95.

21. Rabøl LI, Østergaard D, Mogensen T. Outcomes of classroom-based team training interventions for multiprofessional hospital staff. A systematic review. Qual Saf Health Care 2010; 19:e27.

22. Beckett CD, Kipnis G. Collaborative communication: integrating SBAR to improve quality/patient safety outcomes. J Healthc Qual 2009;31:19-28.

23. Zwarenstein M, Goldman J, Reeves S. Interprofessional collaboration: effects of practice-based interventions on professional practice and healthcare outcomes. Cochrane Database Syst Rev 2009;(3):CD000072.

24. van der Lee N, Driessen EW, Houwaart ES, et al. An examination of the historical context of interprofessional collaboration in Dutch obstetrical care. J Interprof Care 2014;28:123-7.

25. Offerhaus PM, Hukkelhoven CWPM, de Jonge A, et al. Persisting rise in referrals during labor in primary midwife-led care in the Netherlands. Birth 2013:40:192-201.
26. Stichting Perinatale Registratie Nederland. [Dutch Perinatal Registry]. 10 jaar Perinatale Registratie Nederland, de grote lijnen [10 years perinatal registration in the Netherlands, an overview]. Utrecht, 2011.

27. Statistics Netherlands. Bevolkingsontwikkeling [Population growth]. http://statline.cbs.nl/Statweb/publication/?VW=T\&DM=SLNL\&PA= 37230ned\&HD=150313-1001 (accessed Mar 2015)

28. Statistics Netherlands. Geboorte kerncijfers [Birth rates]. http:// statline.cbs.nl/StatWeb/publication/?VW=T\&DM=SLNL\&PA= 37201\&D1=0,2-3\&D2=0-16\&D3=0,I\&HD=090616-1023\&HDR=T, G2\&STB $=$ G1 (accessed Mar 2015).

29. ZonMw Dutch Organisation for Health Research and Development Zwangerschap en Geboorte-Algemeen [Pregnancy and Childbirth] http://www.zonmw.nl/nl/programmas/programma-detail/ zwangerschap-en-geboorte/algemeen/ (accessed Nov 2014).

30. Hussey MA, Hughes JP. Design and analysis of stepped wedge cluster randomized trials. Contemp Clin Trials 2007; 28:182-91.

31. Brown CA, Lilford RJ. The stepped wedge trial design: a systematic review. BMC Med Res Methodol 2006;6:54.

32. Mdege ND, Man MS, Taylor Nee Brown CA, et al. Systematic review of stepped wedge cluster randomized trials shows that design is particularly used to evaluate interventions during routine implementation. J Clin Epidemiol 2011;64:936-48.

33. Salas E, Almeida SA, Salisbury M, et al. What are the critical success factors for team training in health care? Jt Comm J Qual Patient Saf 2009;35:398-405.

34. Chaillet N, Dubé E, Dugas M, et al. Evidence-based strategies for implementing guidelines in obstetrics: a systematic review. Obstet Gynecol 2006;108:1234-45.

35. Mann S, Pratt S, Gluck P, et al. Assessing quality in obstetrical care: development of standardized measures. Jt Comm J Qual Patient Saf 2006;32:497-505

36. Nielsen PE, Goldman MB, Mann S, et al. Effects of teamwork training on adverse outcomes and process of care in labour and delivery: a randomized controlled trial. Obstet Gynecol 2007:109:48-55.

37. Barr $\mathrm{H}$, Freeth $\mathrm{D}$, Hammick $\mathrm{M}$, et al. Evaluations of interprofessional education: a United Kingdom review for health and social care. London, UK: Centre for the Advancement of Interprofessional Education and British Educational Research Association, 2000

38. Sexton JB, Helmreich RL, Neilands TB, et al. The safety attitudes questionnaire: psychometric properties, benchmarking data and emerging research. BMC Health Serv Res 2006;6:44.

39. Kenaszchuk $C$, Reeves $S$, Nicholas $D$, et al. Validity and reliability of a multiple-group measurement scale for interprofessional collaboration. BMC Health Serv Res 2010;10:83.

40. van der Kooy J. Dutch perinatal system: performance and innovative strategies. Erasmus University Rotterdam, 2007.

41. Brown C, Hofer T, Johal A, et al. An epistemology of patient safety research: a framework for study design and interpretation. Part 3End points and measurement. Qual Saf Health Care 2008;17:170-7.

42. Brown C, Hofer T, Johal A, et al. An epistemology of patient safety research: a framework for study design and interpretation. Part 2Study design. Qual Saf Health Care 2008;17:163-9.

43. Craig $\mathrm{P}$, Dieppe $\mathrm{P}$, Macintyre $\mathrm{S}$, et al. Developing and evaluating complex interventions: the new Medical Research Council guidance. BMJ 2008;337:a1655

44. Cunningham NJ, Weiland TJ, van Dijk J, et al. Telephone referrals by junior doctors: a randomised controlled trial assessing the impact of SBAR in a simulated setting. Postgrad Med $J$ 2012;88:619-26. 